\title{
EDITORIAL
}

\section{Automatically controlled continuous positive airway pressure. A bright past, a dubious future}

\author{
D.O. Rodenstein
}

Before the scientific medical community became aware that obstructive sleep apnoea was an extremely prevalent disorder, Suluivan et al. [1] described what is still the best treatment available for this disorder: continuous positive airway pressure (CPAP) applied through the nose during sleep.

The common endpoint of obstructive sleep apnoea, obstructive sleep hypopnoea and upper airway resistance syndrome is an increase in pharyngeal resistance occurring during sleep, leading to increased efficient or inefficient respiratory efforts that usually terminate with an arousal reaction [2]. When respiratory efforts are inefficient, hypoxia and hypercapnia develop during the period of increased pharyngeal resistance until sleep is interrupted, whereas, when respiratory efforts are efficient, hypoxia and hypercapnia are avoided. Nevertheless, the extraordinary effort necessary to sustain ventilation also leads to an arousal reaction [3].

Nasal CPAP acts essentially as a pneumatic splint pushing apart the walls of the pharynx and allowing normal spontaneous ventilation by the patients. In spite of its cumbersome nature, the treatment is so efficient that up to $80 \%$ of patients to whom it is proposed accept and comply with treatment for many years [4]. However, side-effects are not uncommon, and some patients discontinue therapy as time goes by.

The continuous positive pressure necessary to ensure a good nights sleep varies from patient to patient in an individual and poorly predictable way. This makes the individual titration of pressure necessary. Usually this is done during a full or split polysomnographic night, using a trial and error process in which the pressure is slowly increased until apnoeas, hypopnoeas, snoring, episodes of flow limitation and/or arousals are abolished. Drops in saturation may persist when they are due to obesity in addition to sleep apnoea. In such cases, correction of apnoeas does not completely normalize oxygen saturation during the night. The means of titrating the pressure varies from centre to centre and there is no unique well-accepted way of performing titration. In the early years of CPAP, suppression of apnoeas was the main end-point of titration; hypopnoeas came later, and snoring, flow limitation and arousals were incorporated as targets for abolition in later years [5].

The advent of microelectronics and computer power made it possible to find a means of controlling the positive

Correspondence: D.O. Rodenstein, Cliniques universitaires Saint-Luc, Av. Hippocrate 10, 1200 Brussels, Belgium. Fax: 3227643703. pressure delivered by CPAP devices almost instantaneously. The first description of such an automatically controlled CPAP (auto-CPAP) device was published in 1993 [6], and the first clinical applications 2 yrs later [7, 8]. These devices can react extremely rapidly, almost instantaneously, to a series of predetermined signals (events) which may vary from machine to machine. Some machines react to apnoeas, or hypopnoeas, others to snoring and still others to flow limitation or a combination of events. These devices, of course, incorporate means for the detection of apnoeas, hypopnoeas, snoring or flow limitation. This allows the devices to be used in the so-called "diagnostic mode", in which the device identifies and records (but does not react to) these various abnormalities during sleep. In the therapeutic or titration modes, the auto-CPAP devices increase the pressure rapidly when an event is detected, and then decrease it more slowly until a minimum is reached or a new event is detected.

The potential applications of auto-CPAP with its incorporated computer power have been generally assumed to be three-fold. 1) If used in the diagnostic mode, auto-CPAP would obviate the need for cumbersome diagnostic polysomnography, with its time-consuming reading process resulting in high costs and excessive waiting lists [8]. 2) If used to titrate automatically the positive pressure needed, in order to identify a fixed pressure that could then be administered at home via the simpler CPAP devices, it would eliminate the need for titration polysomnography with its high costs in terms of manpower and time [9]. 3) If used for long-term treatment at home, it would allow for high levels of compliance, by avoiding unnecessary excessively high positive pressure resulting from the classic manual titration, given the known or suspected nightto-night changes in the critical pressure responsible for pharyngeal closure. By adapting instantaneously to the needs of the patients, auto-CPAP would yield lower pressures, decreases in side-effects and better compliance [10, $11]$.

Following classical market economy laws, each manufacturer has developed their own auto-CPAP system. The most recent result from the forced oscillation mechanics field $[12,13]$, and are based on continuous monitoring of upper airway resistance by forced oscillations incorporated into the devices that allow for adjustment of pressure to minimize resistance. These machines are currently the object of clinical evaluation, and the number of articles reporting on auto-CPAC increases regularly $[14,15]$. Recent reviews have summarized existing knowledge $[16,17]$. 
When the data presented in these publications is analysed, the following conclusions can be drawn. 1) The promise that auto-CPAP would result in increased compliance has not been fulfilled. Only two articles from the same group report on higher compliance with auto-CPAP than with CPAP $[10,11]$. Later reports denied this increase in compliance [18]. 2) The accuracy of auto-CPAP machines in diagnosing sleep-related disordered breathing is less than it was supposed to be. Signal quality is one problem. The recognition of sleep is another. Many such devices react by increasing the pressure when they detect "hypopnoeas" which are simply due to people being awake and changing their tidal volume [19]. Moreover, snoring and flow limitation during sleep are present in many normal subjects; auto-CPAP devices reacting to snoring and flow limitation without pathological content would unnecessarily "overtreat" these events. 3) Some (though not all [20]) publications report that the median pressure throughout the night is decreased when using auto-CPAP machines $[21,22]$. There is no doubt that this may be true. However, the peak pressure may be higher with automatic devices than with CPAP, for the same long-term results in terms of compliance. 4) When used in the titration mode, the recommended pressure at the end of the titration night may be higher than that obtained when the titration is done manually. 5) Finally, the number of residual events seems to be strictly the same with both types of device during both single night and longterm home use.

All of these points are clearly demonstrated in the study of TESCHLER et al. [23] in this issue of the European Respiratory Journal. TESCHLER et al. [23], in a well designed randomized cross-over protocol, compared manual titration of positive pressure and auto-CPAP automatic titration, followed by long-term home use of either a fixed CPAP (according to the manual titration) or the auto-CPAP device used in the therapeutic mode (i.e. pressure allowed to fluctuate during the night). They found that the compliance was strictly the same, the number of residual events during titration (or repeatedly recovered from the machines at home) were similar for the two devices; and the peak pressures were higher (and the median pressure was lower) with the auto-CPAP than with the CPAP device. Finally, the recommended pressure was significantly higher with autoCPAP than with manual titration.

Thus, after several clinical studies, what remains of the prophecies regarding auto-CPAP value? Three aspects must be considered: 1) auto-CPAP in the diagnostic mode (to replace polysomnography); 2) auto-CPAP to titrate a fixed pressure (to replace the technician); and 3) auto-CPAP for long-term home use (to replace CPAP).

Auto-CPAP in the diagnostic mode is just one of several "limited signal" devices variously used to screen or diagnose sleep-related disordered breathing. The justification for their use varies from "long waiting lists" to "cost considerations". The debate is not closed, and the conclusions (regarding whether limited signal devices are accurate enough to replace polysomnography in a given set of patients with a given clinical level of suspicion) are not clearcut. Economic (cost utility) simulation seems to go against what might seem obvious at first sight by showing that the contribution of polysomnography is not less cost-effective than that of many of the usual medical interventions [24]. Moreover, as more studies are published, the limitations of limited signal devices seem to become more evident, especially those due to mouth breathing and the lack of identification of wakefulness. There is as yet not enough evidence to conclude that auto-CPAP has proved its usefulness in the diagnostic mode.

In the titration mode, auto-CPAP spares technician time, effort and cost. However, there is not as yet a good and proven means of extracting, from a continuously fluctuating pressure signal, a fixed pressure to use at home with a nonautomatic device for long-term use. If the most frequently recommended method (in which the fixed pressure is that that controls sleep-related disordered breathing for $95 \%$ of the time, i.e. is exceeded for only $5 \%$ of the time) is considered, then the pressure may be higher (not lower) than the manually determined one. Thus the main "advantage" of auto-CPAP (decreasing median pressure) is lost. As far as technician costs are concerned, the cost spared on a technician might well be paid back in excess by decreased compliance. Some indications for this come from a report showing a decrease in compliance when a diagnostic procedure was carried out at home rather that in a hospital [25], or from another report showing an increase in compliance when extra time (and effort) was invested at the time of diagnosis [26].

The advantages of long-term home use of auto-CPAP as a treatment device are no better demonstrated. First, autoCPAP devices are more expensive than simpler CPAP machines. Secondly, they do not offer better compliance. Compliance seems to depend more on management strategy, symptoms improvement and psychological factors than on the sophistication of the device. Thirdly, the socalled "advantage" of auto-CPAP (lower median pressure) is balanced out by the "disadvantage" (higher peak pressure) without decreasing residual events.

In conclusion, as the article by TESCHLER et al. [23] nicely shows, it seems extremely hard, by adding technical sophistication, to improve long-term acceptance of and compliance with CPAP therapy in the general patient with sleep apnoea. This may well be because the simple and robust treatment devised by SulLIVAN et al. [1] in 1981 is so efficient that it is just (almost) impossible to improve on it.

Is there a future for auto-continuous positive airway pressure? Even if the scientific and clinical evaluations to date show no added value from auto-continuous positive airway pressure, marketing considerations and the attraction of "novelty" may decide otherwise. There is perhaps a limited group of patients that might derive benefit from therapeutic auto-continuous positive airway pressure. However, this potential limited group has not yet been identified. Now seems the right time to stop evaluating auto-continuous positive airway pressure devices against continuous positive airway pressure in the general patient with sleep apnoea, and to begin the search for the right patient for auto-continuous positive airway pressure. If this potential group is not found, or does not exist, the bright prophecies regarding auto-continuous positive airway pressure will become no more real in the future than they have to date.

\section{References}

1. Sullivan CE, Issa FG, Berthon Jones M, Eves L. Reversal of obstructive sleep apnea by continuous positive airway 
pressure applied through the nares. Lancet 1981; i: $862-$ 865.

2. Collard P, Dury M, Delguste P, Aubert G, Rodenstein DO. Movement arousals and sleep-related disordered breathing in adults. Am J Respir Crit Care Med 1996; 154: 454 459.

3. Zamagni M, Sforza E, Boudewijns A, Petiau C, Krieger J. Respiratory effort. A factor contributing to sleep propensity in patients with obstructive sleep apnea. Chest 1996; 109: 651-658.

4. Collard P, Pieters T, Aubert G, Delguste P, Rodenstein DO. Compliance with nasal CPAP in obstructive sleep apnea patients. Sleep Med Rev 1997; 1: 33-44.

5. Montserrat JM, Ballester E, Olivi H, et al. Time-course of stepwise CPAP titration. Behavior of respiratory and neurological variables. Am J Respir Crit Care Med 1995; 152: $1854-1859$.

6. Berthon-Jones M. Feasibility of a self-setting CPAP machine. Sleep 1993; 16: S120-S121.

7. Gugger M, Mathis J, Bassetti C. Accuracy of an intelligent CPAP machine with in-built diagnostic abilities in detecting apneas: a comparison with polysomnography. Thorax 1995; 50: 1199-1201.

8. Bradley PA, Mortimore IL, Douglas NJ. Comparison of polysomnography with ResCare Autoset in the diagnosis of the sleep apnea/hypopnoea syndrome. Thorax 1995; 50: $1201-1203$

9. Series F. Auto CPAP in the treatment of sleep apnea hypopnea syndrome. Sleep 1996; 19: S281-S283.

10. Meurice JC, Marc I, Series F. Efficacy of auto-CPAP in the treatment of obstructive sleep apnea/hypopnea syndrome. Am J Respir Crit Care Med 1996; 153: 794-798.

11. Series F, Marc I. Efficacy of automatic continuous positive airway pressure therapy that uses an estimated required pressure in the treatment of the obstructive sleep apnea syndrome. Ann Intern Med 1997; 127: 588-595.

12. Farre R, Peslin R, Rotger M, Navajas D. Inspiratory dynamic obstruction detected by forced oscillation during CPAP. A model study. Am J Respir Crit Care Med 1997; 155: 952-956.

13. Farre R, Rotger M, Montserrat JM, Navajas D. A system to generate simultaneous forced oscillation and continuous positive airway pressure. Eur Respir $J$ 1997; 10: 1349-1353.

14. Badia JR, Farre RO, John KR, et al. Clinical application of the forced oscillation technique for CPAP titration in the sleep apnea/hypopnea syndrome. Am J Respir Crit Care Med 1999; 160: 1550-1554.

15. Randerath WJ, Parys K, Feldmeyer F, Sanner B, Ruhle $\mathrm{KH}$. Self-adjusting nasal continuous positive airway pressure therapy based on measurement of impedance: a comparison of two different maximum pressure levels. Chest 1999; 116: 991-999.

16. Loube DI. Technologic advances in the treatment of obstructive sleep apnea syndrome. Chest 1999; 116: 14261433.

17. Krieger J. Therapeutic use of auto-CPAP. Sleep Med Rev 1999; 3: 159-174.

18. Ficker JH, Wiest GH, Lehnert G, Fischer CJ, Katalinic A, Hahn EG. Auto-CPAP treatment in obstructive sleep apnea syndrome: a prospective randomized study during initiation of treatment. Dtsch Med Wochenschr 1997; 122: 1482-1488.

19. Rees K, Wraith PK, Berthon-Jones M, Douglas NJ. Detection of apneas, hypopnoeas and arousals by the AutoSet in the sleep apnea/hypopnoea syndrome. Eur Respir $J$ 1998; 12: 764-769.

20. Ficker JH, Wiest GH, Lehnen G, Wiest B, Hahn EG. Evaluation of an auto-CPAP device for treatment of obstructive sleep apnea. Thorax 1998; 53: 643-648.

21. Teschler H, Berthon-Jones M. Intelligent CPAP systems: clinical experience. Thorax 1998; 53: S49-S54.

22. Sharma S, Wali S, Pouliot Z, Peters M, Neufeld H, Kryger M. Treatment of obstructive sleep apnea with a self-titrating continuous positive airway pressure (CPAP) system. Sleep 1996; 19: 497-501.

23. Teschler H, Wessendorf TE, Farhat AA, Konietzko N, Berthon-Jones M. Two months auto-adjusting versus conventional nCPAP for obstructive sleep apnoea syndrome. Eur Respir J 2000; 15: 990-995.

24. Chervin RD, Murman DL, Malow BA, Totten V. Costutility of three approaches to the diagnosis of sleep apnea: polysomnography, home testing, and empirical therapy. Ann Intern Med 1999; 130: 496-505.

25. Krieger J, Sforza E, Petiau C, Weiss T. Simplified diagnostic procedure for obstructive sleep apnea syndrome: lower subsequent compliance with CPAP. Eur Respir $J$ 1998; 12: 776-779.

26. Hoy CJ, Vennelle M, Kingshott RN, Engleman HM, Douglas NJ. Can intensive support improve continuous positive airway pressure use in patients with the sleep apnea/hypopnea syndrome? Am J Respir Crit Care Med 1999; 159: 1096-1100. 